\title{
Reusable, adhesiveless and arrayed in-plane microfluidic interconnects
}

\author{
R Lo ${ }^{1}$ and $\mathbf{E}$ Meng \\ Department of Biomedical Engineering, University of Southern California, 149 Commonwealth Drive, \\ Menlo Park, CA 94025, USA \\ E-mail: ellis.meng@usc.edu
}

Received 10 September 2010

Published 28 April 2011

Online at stacks.iop.org/JMM/21/054021

\begin{abstract}
A reusable, arrayed interconnect capable of providing multiple simultaneous connections to and from a microfluidic device in an in-plane manner without the use of adhesives is presented. This method uses a 'pin-and-socket' design in which an SU-8 anchor houses multiple polydimethysiloxane septa (the socket) that each receive a syringe needle (the pin). A needle array containing multiple commercially available $33 \mathrm{G}(203 \mu \mathrm{m}$ outer diameter) needles (up to eight) spaced either 2.54 or $1 \mathrm{~mm}$ (center-to-center) pierces the septa to access the microfluidic device interior. Finite element modeling and photoelastic stress experiments were used to determine the stress distribution during needle insertion; these results guided the SU-8 septa housing and septa design. The impact of needle diameter, needle tip style, insertion rate and number of needles on pre-puncture, post-puncture and removal forces was characterized. Pressurized connections to SU-8 channel systems withstood up to $62 \mathrm{kPa}$ of pressurized water and maintained $25 \mathrm{kPa}$ of pressurized water for over $24 \mathrm{~h}$. The successful integration and functionality of the interconnect design with surface micromachined Parylene $\mathrm{C}$ microchannels was verified using Rhodamine B dye. Dual septa systems to access a single microchannel were demonstrated. Arrayed interconnects were compatible with integrated microfluidic systems featuring electrochemical sensors and actuators.
\end{abstract}

(Some figures in this article are in colour only in the electronic version)

\section{Introduction}

Micro total-analysis systems ( $\mu$ TAS) and lab-on-a-chip (LOC) devices, which integrate microfluidic components such as microchambers, microchannels, pumps and valves, enable improved chemical and biological analyses. Miniaturization offers unique advantages such as laminar flow, reduction in reagent/sample volumes, higher throughput and shorter analysis time. Furthermore, devices with precisely controlled dimensions can be batch fabricated. Given these advantages, microfluidics enjoy widespread popularity in applications such as chemical synthesis, genetic analysis, drug screening and single cell/molecule analysis $[1,2]$.

Microfluidic devices require a reliable means to introduce and access the fluids or biological materials to and from microscopic channels. Reliable microfluidic connectors, or interconnects, however, remain a challenge despite decades

1 Now with Exponent, Inc. of microfluidics research. The lack of reliable microfluidic connector options severely limits practical implementation and adoption of many microfluidic technologies. Standardized macro-to-micro fluidic interconnects, that are batch fabricated, have high assembly yield, have a small interface footprint relative to the overall device layout, and are compatible with commonly used materials and device layouts are still under development.

Fluidic interconnects, to date, are predominantly custom solutions and typically their design process commences only after the design and fabrication of the microfluidic device are complete. Few commercially available solutions exist and even so, these interconnects are large relative to the overall device size. Thus, the overall device footprint must often be increased to accommodate the large interconnects. Current fluidic interconnect methods may require precision alignment, adhesives to secure the interconnect, additional fabrication steps and extensive manual assembly [3, 4]. Most 


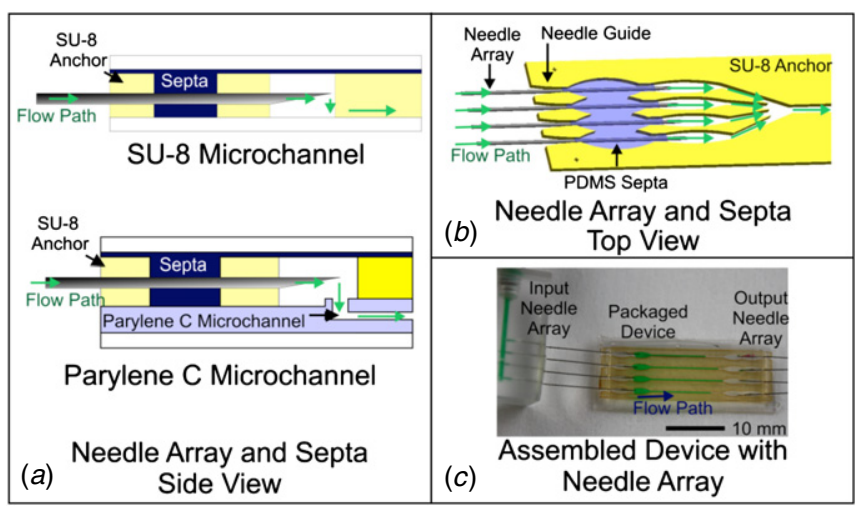

Figure 1. (a) Side view showing concept of single or arrayed microfluidic interconnects. A needle pierces septum to access a microchamber and microchannel in both SU-8 and Parylene C microfluidic devices. (b) Top view schematic showing a needle array piercing septa to allow multiple macro-to-micro connections to be established in an SU-8 microfluidic device. (c) Photograph of an arrayed interconnect mated with an SU-8 microfluidic system.

interconnects are oriented perpendicularly relative to the direction of fluid flow (out-of-plane) in the device which may result in increased dead volume or trapped air pockets [5-13]. Interconnects assembled using adhesives lack robustness and mechanical strength due to the limited bond contact area. Clogs resulting from adhesive flow may also occur and reduce overall yield. Most interconnects are intended for single application/use and cannot be removed without damage to the device, such as the creation of unwanted leakage paths [5, 7-11, 13-16]. Establishing microfluidic connections to surface micromachined microchannels is particularly difficult. Connections are made either by directly interfacing to the channel from the top side or by etching access ports for backside connection. In either case, adhesive or clamped connections require precision alignment and may damage delicate components.

We previously presented an in-plane interconnect using a 'pin-and-socket' approach in which a single commercially available non-coring needle (33G) accessed a microfluidic device by puncturing a polydimethysiloxane (PDMS) septum (figure 1(a)) [17]. The septa shape and location were defined using an SU-8 anchor patterned on a glass substrate. This approach was only demonstrated in simple, single channel SU-8 microfluidic devices.

Practical application of this interconnection approach to broad microfluidic technologies requires a robust method for establishing multiple fluidic interconnections at once. Thus, an arrayed interconnection method for simultaneous connections to multi-channel systems is presented here (figures $1(b)$ and (c)) [18]. Several arrayed interconnects with different spacing (2.54 and $1 \mathrm{~mm}$ ) and septa shape (oval, rectangular and overlapping-ovals) were fabricated and evaluated based on our original concept. Design modifications were confirmed through simulations and photoelastic stress measurements. Needle array insertion forces were experimentally determined. Furthermore, robust fluidic connections were demonstrated with these interconnects under pressurized conditions in both

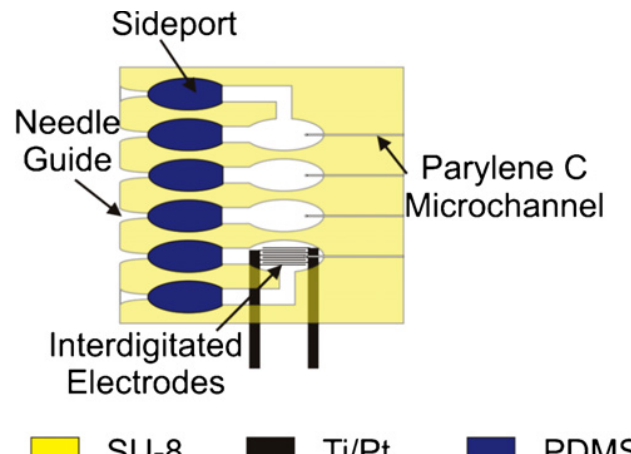

Figure 2. Schematic indicating key features of our interconnect technology and microfluidic system. Here, interconnects with surface micromachined Parylene channels are shown. Needle guides align the needle arrays with the septa. Optional features include sideports and interdigitated electrodes for electrolysis actuation or electrochemical sensing.

SU-8 microchannels and surface micromachined Parylene microfluidic devices.

\section{Device design}

\subsection{Overall design}

The arrayed interconnect uses the pin-in-socket approach, similar to that of the single interconnect [17]. Based on our earlier work, interconnect design was improved to increase the packing density. The high-density design allows multiple needles to simultaneously access corresponding microfluidic channels via their integrated septa without the use of adhesives. Septa were fabricated by filling an SU-8 housing with PDMS prepolymer that was subsequently cured. Then, an array of non-coring needles was inserted through the septa to establish a fluidic connection. PDMS was selected as the septa material for its compliant and resealing properties. The septa formed seals around the needles following insertion and to themselves following removal, preventing unwanted fluid leakage. Several versions of the arrayed interconnect were fabricated to demonstrate the versatility of the technique. Differing septa shapes (oval, oval overlap, rectangular) were investigated; the septa shape used determined the septa spacing ( 2.54 or $1 \mathrm{~mm}$ center-to-center spacing). Additional features unique to the arrayed design were integrated including (1) merged septa for higher-density interconnects, (2) dual septa access to a single channel in the form of a primary access port and sideport, (3) needle guides to align the needle to the septum centers, and (4) lithographically-defined SU-8 or surface micromachined Parylene microchannels (figure 2). Electrochemical transducers (interdigitated electrodes) were also integrated into the microchambers and microchannels.

Oval, overlapped and rectangular septa housing shapes were chosen for their ability to retain PDMS without additional anchoring features (figure 3). We previously presented the relationship between septa design and pull-out force [17]. The theoretical equations and experimental results verified that pull-out force increased linearly with the contact surface 


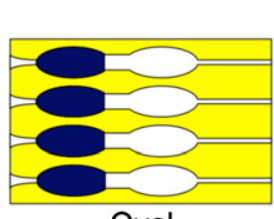

Oval

\section{Septa Shapes}

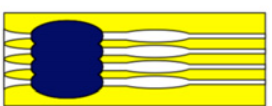

Oval Overlap

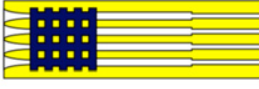

Rectangular SU-8 PDMS

Figure 3. Septa configurations used in the arrayed interconnect designs.

area between the septa and needle. Septa shape did not affect the pull-out force; however, designs which included a septa locking feature (e.g. a shape which prevented the septa from being dislodged from the housing during needle insertion/removal) were preferred.

The oval septa design contains several distinct septa on $2.54 \mathrm{~mm}$ center-to-center spacing. Septa housings were individually filled with PDMS. Overlapped and rectangular septa feature denser interconnect packing (1 mm spacing) and simultaneous filling of multiple septa housings as they are fluidically connected. The thickness of the SU-8 septa housing was determined by the practical fabrication limits for thick planar SU-8 layers (time and process complexity) and the needle outer diameter. For $33 \mathrm{G}$ non-coring needles (203 $\mu \mathrm{m}$ OD), a $300 \mu \mathrm{m}$ thick layer of SU-8 was used.

Septa spacing, and consequently, the density of the connections were determined by the dimensions of the microfluidic channels and integrated components, as well as the septum shape. Two spacing arrangements were considered. A $2.54 \mathrm{~mm}$ center-to-center spacing design utilized separated oval septa. This spacing was chosen to emulate the standard spacing found in electrical pin packages. Denser interconnects with $1 \mathrm{~mm}$ center-to-center spacing were fabricated using overlapping oval septa and connected rectangular septa. Sideports, as well as converging microchannel designs, that allow multiple fluids to be introduced into a single input or channel, respectively, were also investigated.

The angle of needle insertion is important for proper alignment and to prevent interconnect failure. Misalignment may cause the needle to veer off-center during insertion and become lodged against the SU-8 anchor walls. Misalignment in our previous interconnects resulted in blockage of the needle lumen and fluid path [17]. This improved interconnect incorporates needle alignment structures in the SU-8 housing, which direct the needle through the center of the septum.

The microchannels in the arrayed interconnect design were fabricated using SU-8 or Parylene. The previously presented single interconnect design featured an SU-8 channel that was fabricated simultaneously with the SU-8 septa housing using the same photomask. However, the height of the SU-8 microchannel was determined by the thickness of the SU-8 layer; furthermore, the width of the microchannel was also limited based on the manufacturer's recommended aspect ratio for SU-8 structures (10:1 height to width ratio, [19]). Therefore, for a $300 \mu \mathrm{m}$ thick SU-8 layer, a minimum channel width of $30 \mu \mathrm{m}$ was used. For applications requiring channels with dimensions independent of those of the SU8 housing, surface micromachined Parylene channels, which are compatible with our septa interconnect technology, can be used. The channel dimensions and Parylene thickness must be carefully selected to prevent the channel from collapsing when the sacrificial photoresist within the channel is removed [20]. The channel width is obtained from the critical length $\left(l_{\text {crit }}\right)$ for preventing the collapse and stiction of cantilevers. It is governed by the Young's modulus of the channel material $(E)$, the channel wall thickness $(t)$, gravity $(g)$, the surface tension between an air-liquid interface $\left(\gamma_{\text {la }}\right)$ and the contact angle of the material $\left(\theta_{c}\right)$ [21]:

$$
l_{\text {crit }}=\left(\frac{3 E t^{3} g^{2}}{16 \gamma_{\mathrm{la}} \cos \theta_{c}}\right) \text {. }
$$

Yao et al determined a maximum distance of $150 \mu \mathrm{m}$ between supporting structures in a $4.5 \mu \mathrm{m}$ thick Parylene beam. In a channel, the channel walls serve as the supporting structure; therefore, a channel width of $100 \mu \mathrm{m}$ and a channel height of $4 \mu \mathrm{m}$ were chosen, eliminating the need for support posts within the channel [20]. Both narrower and wider Parylene channels are also possible with proper design. This microchannel is significantly thinner than the SU-8 microchannel; the Parylene microchannel has 22.5 times less volume than a SU-8 microchannel of equivalent length.

Microfluidic devices with integrated arrayed interconnects with different septa shapes (oval, oval overlap or rectangular), number of septa (4 or 8 ), septa spacing (1 or $2.54 \mathrm{~mm}$ ), microchannel material (SU-8 or Parylene) and optional sideports were designed and fabricated. Some devices also incorporated converging channels and metal structures to demonstrate the versatility of this interconnect technique. A representative arrayed interconnect with Parylene microchannels, oval overlap septa with eight inputs and outputs, sideports and needle guides is shown in figure 4.

\section{Fabrication}

\subsection{SU-8 microchannel without metal}

The fabrication process for arrayed interconnects with SU8 microchannels and no metal features is identical to that previously reported for the single interconnect [17]. In summary, an SU-8 anchor was fabricated using a soda lime wafer substrate (Silicon Quest International, Santa Clara, CA). A $2 \mu \mathrm{m}$ layer of Parylene (Specialty Coating Systems, Inc., Indianapolis, IN) was vapor deposited onto the adhesion promoter treated-wafer (A-174, Specialty Coating Systems, Indianapolis, IN). A $300 \mu \mathrm{m}$ layer of SU-8 (SU-8 2100, Microchem Corp., Newton, MA) was spin coated onto the Parylene using a two-step process. A controlled soft bake using a programmable hotplate was performed following application of each SU-8 layer. The SU-8 was patterned $\left(600 \mathrm{~mJ} \mathrm{~cm}^{-2}\right)$, hardbaked and developed. Septa were created by filling the SU-8 anchor with PDMS. A water masking technique, which was previously demonstrated, was used to prevent the PDMS from spreading outside the septa area $[17,22]$. A half-cured and uncured layer of PDMS was used to secure a glass cap to seal the microfluidic system [23]. 


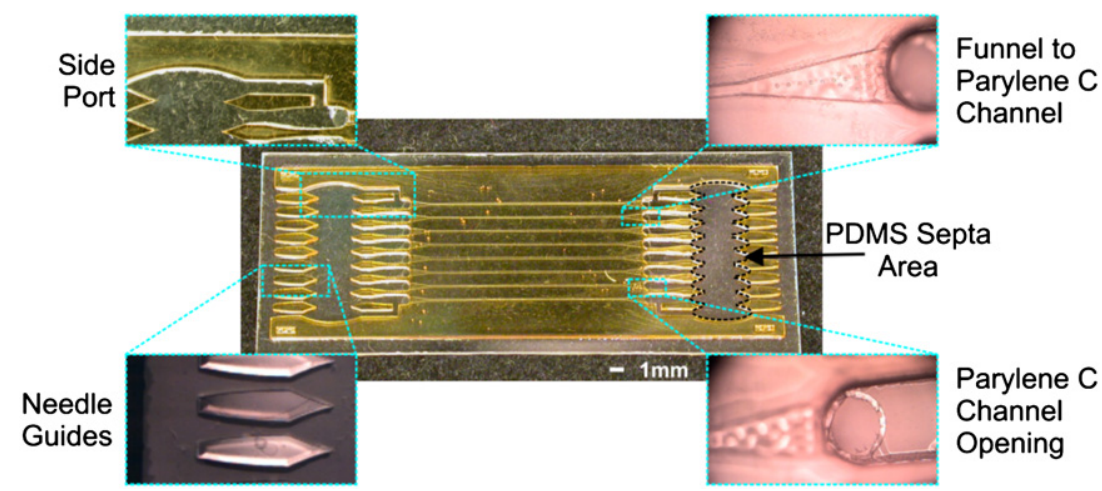

Figure 4. Fabricated arrayed interconnect with Parylene microchannels and sideports. Salient features of the arrayed microfluidic system with integrated interconnects are highlighted. External access provided via needles inserted through the septa is not shown in these photographs.

\subsection{SU-8 microchannel with metal}

Incorporating metal patterns, such as resistive elements and interdigitated electrodes, into the microfluidic device design required additional fabrication steps prior to Parylene deposition. A $4 \mu \mathrm{m}$ layer of AZ 4400 (4 krpm, $40 \mathrm{~s}$ ) was spun onto the wafer surface. The liftoff photoresist pattern was exposed $\left(240 \mathrm{~mJ} \mathrm{~cm}^{-2}\right)$ and developed. Then the wafer was descummed in oxygen plasma $(30 \mathrm{~s}, 60 \mathrm{~W}, 100 \mathrm{mTorr})$ and a layer of Ti/Pt (300 $/ 1000 \AA)$ was electron beam evaporated onto the substrate. Liftoff in acetone revealed the metal patterns. Then a $2 \mu \mathrm{m}$ layer of Parylene was deposited onto the wafer. A masking layer of photoresist (AZ 4400, $4 \mu \mathrm{m}$, $4 \mathrm{krpm}, 40 \mathrm{~s}$ ) was spun and patterned. Parylene was etched in oxygen plasma $(150 \mathrm{~W}, 100 \mathrm{mTorr}, 10 \mathrm{~min})$ to remove open the contact pads and electrodes. SU-8 was applied as directed in the fabrication steps listed above.

\subsection{Parylene microchannel with metal}

Arrayed interconnect with Parylene microchannels started with Parylene $(2 \mu \mathrm{m})$ coated soda lime wafers. A photoresist etch mask (AZ 4400, 4 krpm, 30 s) was spin coated and patterned. The Parylene layer was etched using oxygen plasma to create contact pad openings. Lithography and metal liftoff were performed as previously mentioned to lay down interdigitated electrochemical electrodes. Sacrificial photoresist (AZ 4400, $4 \mathrm{krpm}, 30 \mathrm{~s}$ ) was applied and patterned to define channels. A second layer of Parylene $(4 \mu \mathrm{m})$ was deposited to form the remaining three walls of the microchannel. The wafer was then baked in vacuum at $160{ }^{\circ} \mathrm{C}$ for $40 \mathrm{~h}$ to anneal the two layers of Parylene [24]. Next, a $10 \mu \mathrm{m}$ photoresist etch mask (AZ 4620, $2 \mathrm{krpm}$, 45 s) was spin coated and patterned to define the channel openings. The Parylene was removed using oxygen plasma. Finally, the SU-8 layer, septa formation and device packaging were completed using the same process steps as described for the SU-8 microchannel design. The sacrificial photoresist defining the Parylene microchannels was dissolved in a room temperature isopropyl alcohol (IPA) bath to prevent adverse interactions to SU-8 structures following exposure to acetone (e.g. SU-8 delamination and cracking). Removal of the

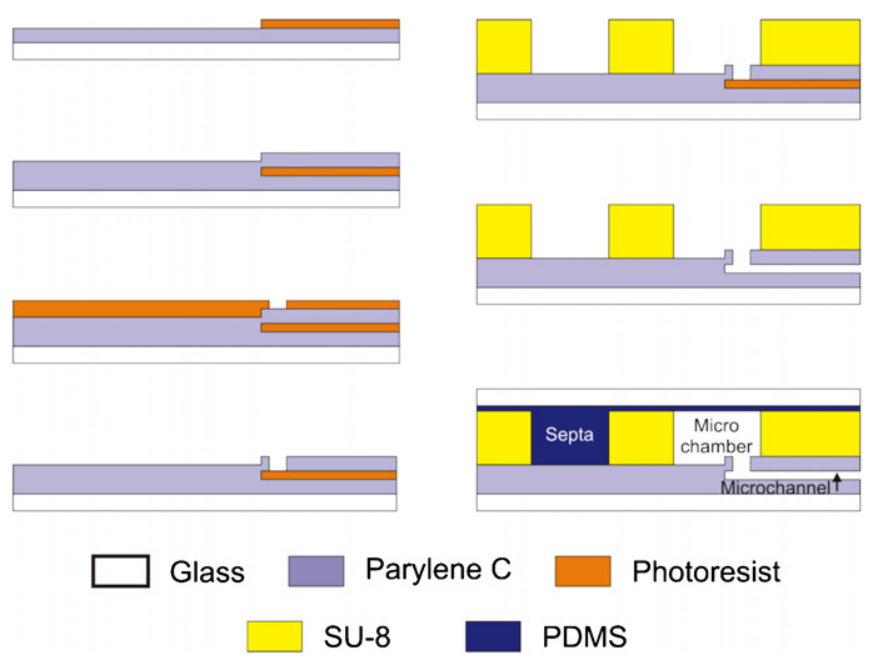

Figure 5. Process steps to fabricate and package an arrayed interconnect with a surface micromachined Parylene microchannel.

sacrificial photoresist can be accelerated using an undiluted photoresist developer. The fabrication process for Parylene microchannels without metal is shown in figure 5 .

\subsection{Needle array}

The needles were housed in channels ( $343 \mu \mathrm{m}$ in diameter) spaced 1 or $2.54 \mathrm{~mm}$ apart, center-to-center. Two different types of needle arrays were fabricated: shared input (figures 6(a)-(c)) and separated inputs (figures 6(d) and $(e)$ ). The shared input needle array was fabricated by drilling channels (\#80 drill bit) partially through a Plexiglas block. A larger diameter channel (\#60 drill bit) was drilled through the side of the block that intersected all the smaller channels. A 10-32 threaded hole allowed connection of a liquid or gas source to the needle array with conventional fittings. Commercially available $33 \mathrm{G}$ non-coring (i.e. beveled tip) needles (21033A Point Style 2, Hamilton Company, Reno, $\mathrm{NV}$ ) were carefully placed in each of the \#80 holes and affixed using epoxy. In this configuration, pressurized media were applied to all the needles simultaneously; however, needle arrays with separate fluidic access to each needle were also fabricated. 


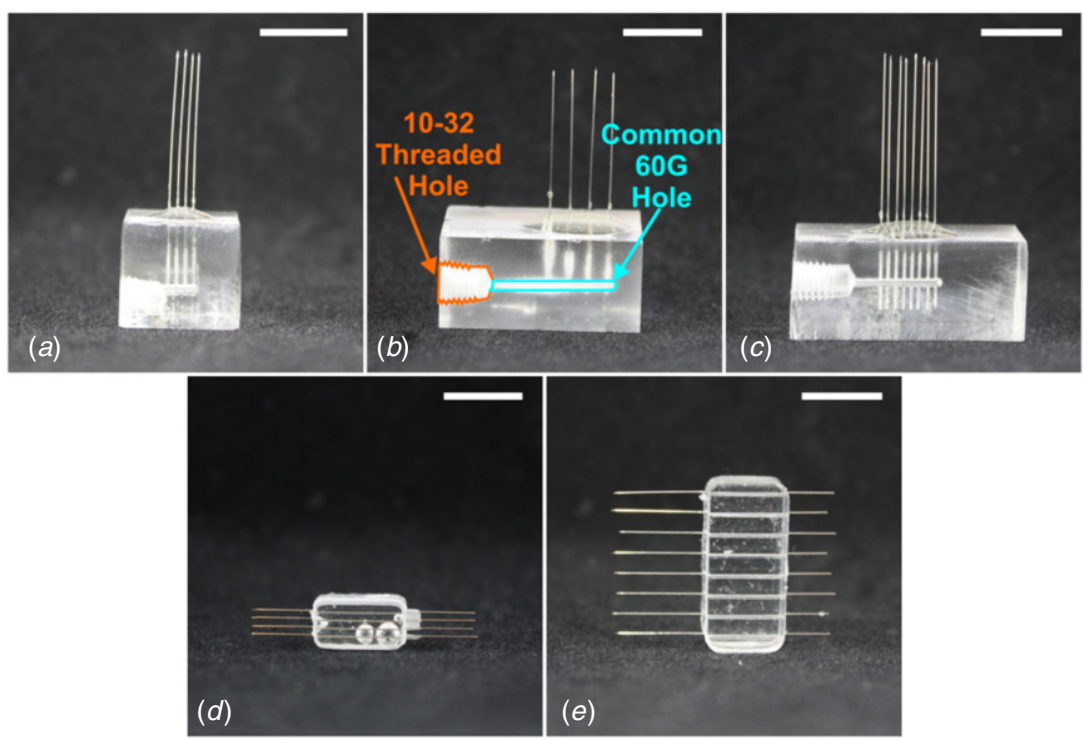

Figure 6. Photographs of needle arrays that provide shared or separate access to microchannels: $(a)$ four shared needles with $1 \mathrm{~mm}$ spacing, (b) four shared needles with $2.54 \mathrm{~mm}$ spacing, $(c)$ eight shared needles with $1 \mathrm{~mm}$ spacing, $(d)$ four separate needles with $1 \mathrm{~mm}$ spacing, and (e) eight separate needles with $2.54 \mathrm{~mm}$ spacing. The scale bar represents $10 \mathrm{~mm}$.

A custom-made Plexiglas mold was used to fabricate a needle array with individual needle access. The needles were placed into grooves in the mold that controlled spacing and alignment. Casting acrylic was poured into the mold to form a monolithic block with embedded needles. Silicone tubes were attached to each needle.

\section{Experimental methods}

\subsection{FEM analysis of stress distribution}

Finite element analysis and modeling estimated the stress on the septa as the needles were inserted. Only the oval overlap septa were modeled; the simple design and ease of fabrication make it a likely candidate for future use. The single septum case had been previously studied for the single interconnect design [25]. Additionally, the oval overlapped model was used because multiple septa were merged into a single PDMS slab; therefore, any insertion stress affecting neighboring septum/needle pairs will be apparent in the model. The stress distribution in the PDMS septa set a practical limit to the achievable needle density by dictating the minimum spacing between the needle path and SU-8 housing. A suggested design rule is a minimum distance between the needle shaft and the SU-8 housing equaling twice the length required to dissipate to $36.8 \%$ (1/e) of the maximum stress value.

The static stress distribution within the septa was modeled at three needle insertion points: (1) as needles touched the surface of the septa, (2) after the needles pierced the septa and are partially inserted, and (3) after needles were fully inserted. Septa surface area adjacent to the housing (e.g. SU-8 anchor, device substrate and device cap) was held constant. The bonding strength between the PDMS and the SU-8 housing or the Parylene coated substrate does not preclude the PDMS from delaminating from either surface; however, debonding between the PDMS and SU-8 or substrate boundaries is a dynamic interaction which is difficult to approximate in the model. The septa faces not in contact with the SU-8 housing, substrate or packing (e.g. faces through which the needle can enter or exit) were not constrained. The insertion force at the needle tip and friction force along the needle shaft were applied using force data obtained experimentally with a Bose 3100 ElectroForce mechanical fatigue test instrument. The modeling results were also compared to photoelastic stress images.

\subsection{Photoelastic stress}

PDMS is a photoelastic material; stresses in the PDMS during needle array insertion and removal can be visualized using polarized light. The principal stresses $\left(\sigma_{x}, \sigma_{y}\right)$ can be visually observed as a phase difference $(\Delta)$ that is dependent on the wavelength $(\lambda)$, the material stress-optical coefficient $(C)$, and material thickness $(h)$ [26]:

$$
\Delta=\frac{2 \pi h}{\lambda} C\left(\sigma_{x}-\sigma_{y}\right) .
$$

PDMS slabs were pierced using a single needle and needle array to visualize resulting stresses. Each slab was placed between two polarizing plates and illuminated with a broadband light source positioned below the stack. The polarizing plates were rotated to an orientation which provided the greatest visible contrast in stressed versus non-stressed areas. Low stress areas appeared as a white haze where higher stress areas exhibited a rainbow effect. Photoelastic images were taken during initial needle puncture and partial needle insertion into the PDMS sample to compare with FEM results.

\subsection{Insertion test}

The force required to insert and remove a single needle or needle array into a PDMS slab (thickness $2 \pm 0.1 \mathrm{~mm}$ ) was 
measured using a Bose 3100 ElectroForce mechanical fatigue test instrument and custom-made laser-cut Plexiglas jigs. The PDMS slab was mounted between two clamped Plexiglas plates. The plates had through holes $(1,4$ or 8 ) spaced $2.54 \mathrm{~mm}$ apart which guided the corresponding needle arrangements through the PDMS slab. The jig was attached to a load cell at the base of the Bose instrument. Needles were lowered at a constant rate and the force required to puncture the PDMS sample (insertion force) and to remove the needles from the sample (pull-out force) was recorded.

Insertion force is a combination of pre-puncture and postpuncture forces. The pre-puncture force, or stiffness force ( $\left.f_{\text {stiffness }}\right)$, is attributed to material deformation. Post-puncture force is a combination of the force required to push a needle through the PDMS slab ( $\left.f_{\text {cutting }}\right)$ and the friction force $\left(f_{\text {friction }}\right)$ between the needle shaft and the PDMS [27, 28]. Thus, the insertion force is expressed as

$$
f_{\text {insertion }}=f_{\text {stiffness }}+f_{\text {cutting }}+f_{\text {friction }} \text {. }
$$

The force required to remove the needle (pull-out force) is a combination of frictional and debonding forces [29]:

$$
f_{\text {pull-out }}=f_{\text {debonding }}+f_{\text {friction }} \text {. }
$$

A discussion of the pull-out force was previously presented for a single interconnect case [17]. In summary, Gent and Liu modeled debonding between a fiber (i.e. the needle) embedded in a matrix (i.e. the septa) using a modified theory based on the Griffith fracture energy criterion [29]. The debonding force is a function of the cross-sectional area of the matrix, fiber radius, the Young's modulus of the matrix and the adhesive fracture energy between the matrix and the fiber. The frictional force varies linearly with respect to the coefficient of friction between the fiber and the matrix, the compressive stress and the contact area between the fiber and the matrix.

The relationship between insertion force and the number of needles (1, 4 or 8$)$, needle type (C: coring, or NC: noncoring), needle gauge $(33,30$ or $27 \mathrm{G})$, and insertion rate $(0.5$ or $1 \mathrm{~mm} \mathrm{~s}^{-1}$ ) was determined. All needles were obtained from Hamilton Company (Reno, NV). Additionally, needle insertion was completed multiple times on a single sample to examine magnitude of the insertion and removal forces with each subsequent use. The two needle tip types used in this experiment are classified as coring (C) or non-coring (NC). The coring needle has a blunt tip which cores a cylindrical section from the material as it is inserted whereas the noncoring needle has a beveled tip and displaces material as the needle is pushed through.

The insertion rates were selected based on the limitations of the Bose 3100 ElectroForce instrument. The maximum stroke distance of the displacement motor is $4 \mathrm{~mm}$. A $1 \mathrm{~mm}$ $\mathrm{s}^{-1}$ rate modeled a practical insertion rate while maintaining a high sample rate resolution from the Bose instrument. A second rate, which was half of the base rate value, was selected for comparison.

\subsection{Pressure test}

Pressurized DI $\mathrm{H}_{2} \mathrm{O}$ was applied to assembled SU-8 microfluidic systems to determine failure pressures and modes.
Failure pressures were obtained for all three septa designs (oval, oval overlap and rectangular) in the four-microchannel configuration. The design with the greatest failure pressure was compared to that of the eight-microchannel device with the same septa design. Needles were inserted into the input and output septa. Dyed DI $\mathrm{H}_{2} \mathrm{O}$ was first introduced into the system to ensure that the microchannel was open and free from obstructions. Then the output needles were removed. Pressure was applied in increments of 0.5 psi $(3.45 \mathrm{kPa})$ with a 5 min hold period to allow system equilibration. The failure pressure was recorded when water leakage was observed.

Prolonged pressure was applied to a device to ensure that the arrayed interconnect can withstand pressure application for an extended period of time. The applied pressure was at least $50 \%$ of the average maximum pressures of all the tested interconnects. The pressure was applied for $24 \mathrm{~h}$ and observed to ensure no leakage during the entire period.

\subsection{Parylene microchannel}

Surface micromachined Parylene microchannels with PDMS septa were also demonstrated. These may be used instead of SU-8 microchannels when finer dimensional control of channels or a Parylene surface is required. Also, complex and highly integrated microfluidic systems are possible only using Parylene surface micromachining technology. Microchannel patency was verified using capillary wicking of diluted Rhodamine B dye into the microchannel opening prior to forming the PDMS septa. Flow of pressurized Rhodamine $\mathrm{B}$ introduced through the inserted needles was monitored by optical microscopy.

\subsection{Electrolysis pressure generation}

Internal pressure was generated using the interdigitated electrodes. The water within the device was converted to hydrogen and oxygen gas following current application (0.3 mA) to the electrolysis electrodes. The internal pressure was monitored (ASDX 015D44R, Honeywell International, Morristown, NJ). A baseline reading of atmospheric pressure was obtained prior to each test. Current was applied to the device until the entire device interior was voided of any visible water (approximately $100 \mathrm{~s}$ ).

\subsection{Sideport functionality}

The dual septa feature with one primary septum and one sideport was demonstrated. $33 \mathrm{G}$ non-coring needles were inserted through the septa of the sideport and main channel. A 30G non-coring needle was inserted into the output port to minimize any fluidic resistance at the output. Syringes containing either deionized water $\left(\mathrm{DI} \mathrm{H}_{2} \mathrm{O}\right.$ ) or dyed DI $\mathrm{H}_{2} \mathrm{O}$ were connected to each input needle. A syringe pump was used to deliver a constant steady flow to both needles. 


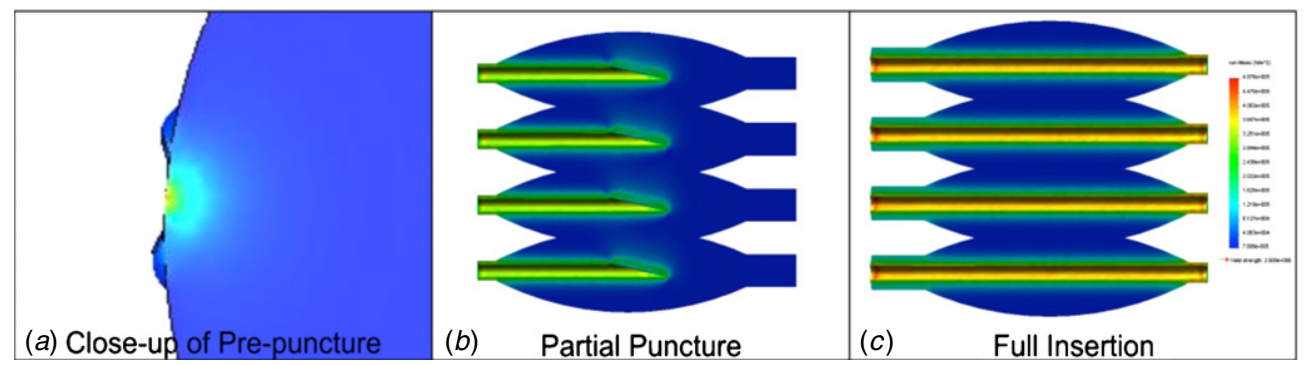

Figure 7. FEM images of stress distribution within septa during needle insertion, $(a)$ pre-puncture, $(b)$ partial puncture, and $(c)$ complete insertion.

\section{Results}

\subsection{FEM analysis of stress distribution}

Finite element modeling of both a single septum and multiple septa designs showed that the stress concentration remained localized around the needle. A radial stress pattern was observed at the needle tip pre-puncture and became asymmetric during insertion, matching the beveled tip of the needle. After insertion, the stress was uniformly distributed along the needle shaft and extended to neighboring septa (figure 7). The maximum stress was observed at the needle/septa interface and decayed exponentially away from the needle. The distance over which the stress decayed to $1.35 \times 10^{5} \mathrm{~N} \mathrm{~mm}^{-2}$ (36.8\% of the maximum stress, $3.63 \times$ $10^{5} \mathrm{~N} \mathrm{~mm}^{-2}$ ) was approximately $60 \mu \mathrm{m}$.

The FEM results modeled the stress distribution for ideal conditions where (1) all needles are aligned within the septa, (2) needles do not deviate from a straight path, (3) needles maintain a uniform distance from one another, (4) all needles are of the same length, (5) all needle tips (e.g. the bevels) are orientated in the same direction, and (6) needle insertion force is only directed parallel to the needle shaft. The resultant stress distribution identified the minimum dimensions necessary for the interconnect design to prevent unwanted stress interference on the SU-8 septa housing or adjacent septum/needle pairs. The data suggest a minimum $240 \mu \mathrm{m}$ spacing between adjacent $33 \mathrm{G}$ needles, indicating that tighter septa packing and thus denser microfluidic interconnects are possible compared to what is demonstrated here.

\subsection{Photoelastic stress}

Larger diameter needles (single needle: $18 \mathrm{G}$, needle array: $27 \mathrm{G}$ ) were necessary to enhance the visible photoelastic stress during needle insertion. Photoelastic stress using $33 \mathrm{G}$ needles was not visible with the limited resolution of our imaging equipment. From the FEM analysis using a $33 \mathrm{G}$ needle, the visible stress within the PDMS extended approximately $100 \mu \mathrm{m}$ from the needle shaft; the recording camera did not have sufficient resolution to detect this distance.

Stress concentrations were visible along the needle shaft and as a plume at the needle tip during insertion. Photoelastic visualization of insertion of a $2.54 \mathrm{~mm}$ spaced needle array demonstrated that the stress distribution from one needle

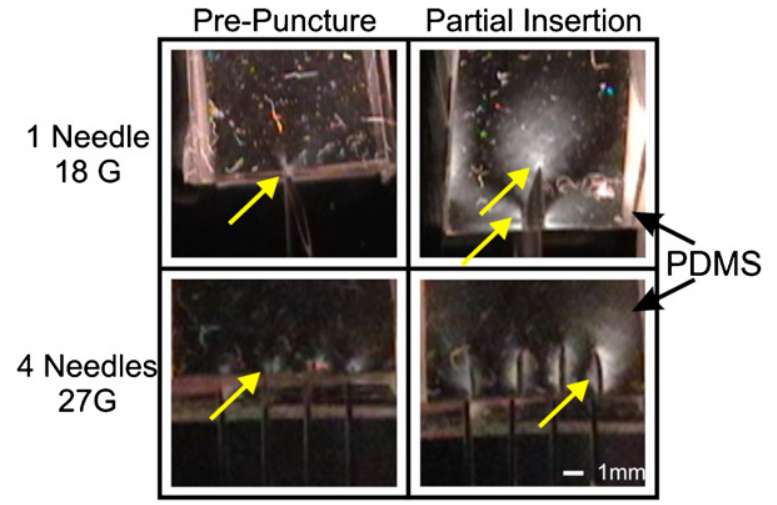

Figure 8. Photoelastic stress in PDMS from needle insertion for a single needle (18G) and needle array (four $27 \mathrm{G}$ ). The arrows indicate visualized photoelastic stress during pre-puncture (when the needle tip touches the PDMS surface) and partial insertion (when the needle has been inserted partially through the PDMS).

shaft did not overlap with that of a neighboring needle. This suggests that stresses from each needle/septum pair do not affect neighboring needle/septum pairs. These results are similar to the FEM prediction for stress distribution (figure 8); however, a direct comparison cannot be made due to the difference in needle size and variation in needle bevel orientation in hand-made needle arrays.

\subsection{Insertion test}

Stiffness, insertion (post-puncture insertion force is a combination of friction and cutting forces) and removal forces were identified for several needle arrangements (table 1). Figure 9 shows a representative graph of the force measurement for a needle array during insertion and removal. The stiffness force was measured by identifying a slight dip in force between the needle touching the PDMS surface (figure $9(a)$ ) and full needle puncture (figure $9(b)$ ). The postpuncture force (cutting and frictional forces) was measured in figure $9(b)$ and the frictional force was measured in figure $9(c)$. The cutting force was calculated by subtracting the frictional force value (figure $9(c)$ ) from the post-puncture force. The maximum pull-out force was measured in figure 9(e). Previous work demonstrated that puncturing the same location multiple times decreased sealing ability of the material, compared to random insertion locations [30]. Therefore, needle 
Table 1. Summary of relationship between insertion and removal forces and the needle type (coring (C) versus non-coring (NC)), needle gauge $(27 \mathrm{G}$ or $33 \mathrm{G})$, number of needles $(1,4$ or 8$)$ and rate of insertion $\left(0.5\right.$ or $\left.1 \mathrm{~mm} \mathrm{~s}^{-1}\right)$ (mean $\left.\pm \mathrm{SE}, n=4\right)$.

\begin{tabular}{|c|c|c|c|c|c|c|c|c|c|}
\hline $\begin{array}{l}\text { Needle } \\
\text { gauge } \\
(\mathrm{OD}(\mu \mathrm{m}))\end{array}$ & $\begin{array}{l}\text { Needle } \\
\text { point } \\
\text { type }\end{array}$ & $\begin{array}{l}\text { No of } \\
\text { needles }\end{array}$ & $\begin{array}{l}\text { Insertion } \\
\text { rate } \\
\left(\mathrm{mm} \mathrm{s}^{-1}\right)\end{array}$ & $\begin{array}{l}\text { No of } \\
\text { insertions }\end{array}$ & $\begin{array}{l}\text { Stiffness } \\
\text { force }(\mathrm{N})\end{array}$ & $\begin{array}{l}\text { Insertion } \\
\text { force }(\mathrm{N})\end{array}$ & $\begin{array}{l}\text { Friction } \\
\text { force }(\mathrm{N})\end{array}$ & $\begin{array}{l}\text { Cutting } \\
\text { force }(\mathrm{N})\end{array}$ & $\begin{array}{l}\text { Removal } \\
\text { force }(\mathrm{N})\end{array}$ \\
\hline 27G (406) & $\mathrm{C}$ & 1 & 1 & 1 & $1.87 \pm 0.14$ & $1.67 \pm 0.16$ & $1.3 \pm 0.11$ & $0.37 \pm 0.07$ & $1.4 \pm 0.2$ \\
\hline 33G (203) & $\mathrm{NC}$ & 1 & 1 & 1 & $0.18 \pm 0.02$ & $0.87 \pm 0.02$ & $0.74 \pm 0.02$ & $0.14 \pm 0.04$ & $0.6 \pm 0.03$ \\
\hline 33G (203) & $\mathrm{C}$ & 1 & 1 & 1 & $0.83 \pm 0.03$ & $1.2 \pm 0.01$ & $0.83 \pm 0.02$ & $0.37 \pm 0.02$ & $0.89 \pm 0.02$ \\
\hline $27 \mathrm{G}(406)$ & $\mathrm{NC}$ & 4 & 1 & 1 & $\mathrm{~N} / \mathrm{A}$ & $5.41 \pm 0.34$ & $5.24 \pm 0.49$ & $0.18 \pm 0.11$ & $2.53 \pm 0.17$ \\
\hline 33G (203) & $\mathrm{NC}$ & 4 & 1 & 1 & N/A & $3.3 \pm 0.05$ & $2.81 \pm 0.06$ & $0.49 \pm 0.04$ & $2.65 \pm 0.07$ \\
\hline 33G (203) & $\mathrm{NC}$ & 4 & 0.5 & 1 & $\mathrm{~N} / \mathrm{A}$ & $3.48 \pm 0.08$ & $3 \pm 0.05$ & $0.49 \pm 0.05$ & $2.7 \pm 0.1$ \\
\hline 33G (203) & $\mathrm{NC}$ & 8 & 1 & 1 & $\mathrm{~N} / \mathrm{A}$ & $5.72 \pm 0.15$ & $4.88 \pm 0.16$ & $0.84 \pm 0.02$ & $4.6 \pm 0.15$ \\
\hline $27 \mathrm{G}(406)$ & $\mathrm{C}$ & 1 & 1 & 9 & N/A & $1.25 \pm 0.09$ & $1.25 \pm 0.09$ & $0 \pm 0$ & $1.11 \pm 0.02$ \\
\hline 33G (203) & $\mathrm{NC}$ & 1 & 1 & 10 & N/A & $0.61 \pm 0.01$ & $0.61 \pm 0.01$ & $0 \pm 0$ & $0.54 \pm 0.03$ \\
\hline 33G (203) & C & 1 & 1 & 9 & $\mathrm{~N} / \mathrm{A}$ & $0.66 \pm 0.01$ & $0.66 \pm 0.01$ & $0 \pm 0$ & $0.71 \pm 0.02$ \\
\hline $27 \mathrm{G}(406)$ & $\mathrm{NC}$ & 4 & 1 & 10 & $\mathrm{~N} / \mathrm{A}$ & $4.67 \pm 0.21$ & $4.67 \pm 0.21$ & $0 \pm 0$ & $2.64 \pm 0.2$ \\
\hline 33G (203) & $\mathrm{NC}$ & 4 & 1 & 10 & N/A & $2.45 \pm 0.08$ & $2.42 \pm 0.07$ & $0.03 \pm 0.03$ & $2.43 \pm 0.09$ \\
\hline 33G (203) & $\mathrm{NC}$ & 4 & 0.5 & 10 & N/A & $2.71 \pm 0.07$ & $2.7 \pm 0.07$ & $0.01 \pm 0$ & $2.49 \pm 0.02$ \\
\hline 33G (203) & $\mathrm{NC}$ & 8 & 1 & 10 & $\mathrm{~N} / \mathrm{A}$ & $4.42 \pm 0.15$ & $4.42 \pm 0.13$ & $0 \pm 0$ & $4.37 \pm 0.12$ \\
\hline
\end{tabular}

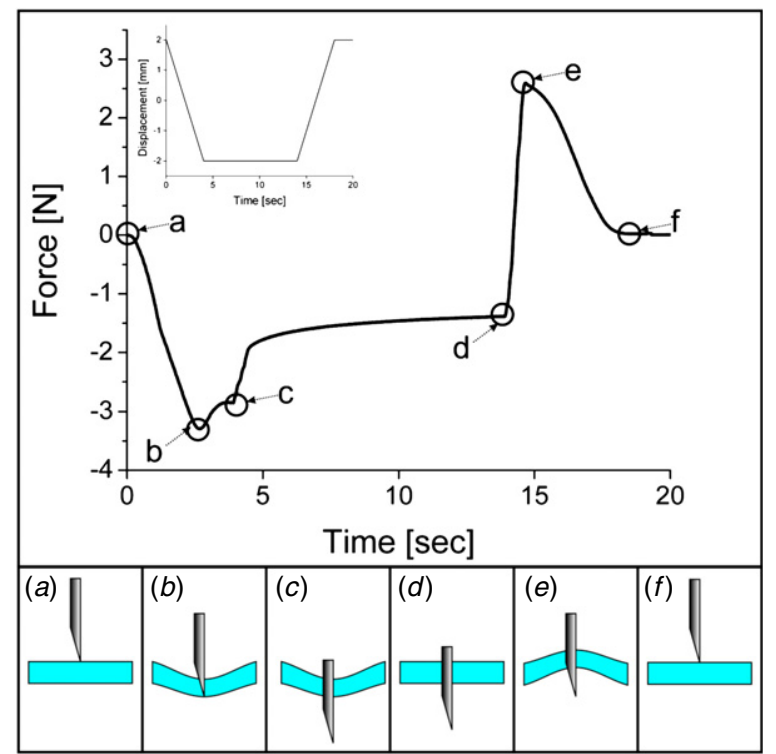

Figure 9. Representative results from insertion force tests: (a) needle touches the surface of the PDMS sample, $(b)$ needle pierces the PDMS sample (combination of stiffness and puncture forces), (c) needle moves through PDMS (friction force), $(d)$ needle stops moving and material relaxes, $(e)$ needle removal from PDMS (max removal force), and $(f)$ needle fully removed. Inset: corresponding needle displacement over time.

insertion and removal cycles were performed at least nine times at the same location in a PDMS slab to determine the effect of multiple punctures on insertion and removal forces.

As predicted from insertion force equations [29], the forces for the $33 \mathrm{G}$ single-, four- and eight-needle array, on average, varied linearly with the number of needles (figure 10). This result is also in agreement with the results presented by Okamura et al where the forces of insertion in soft tissue were presented [28]. Practically speaking, the maximum number of needles that can be simultaneously inserted is limited by

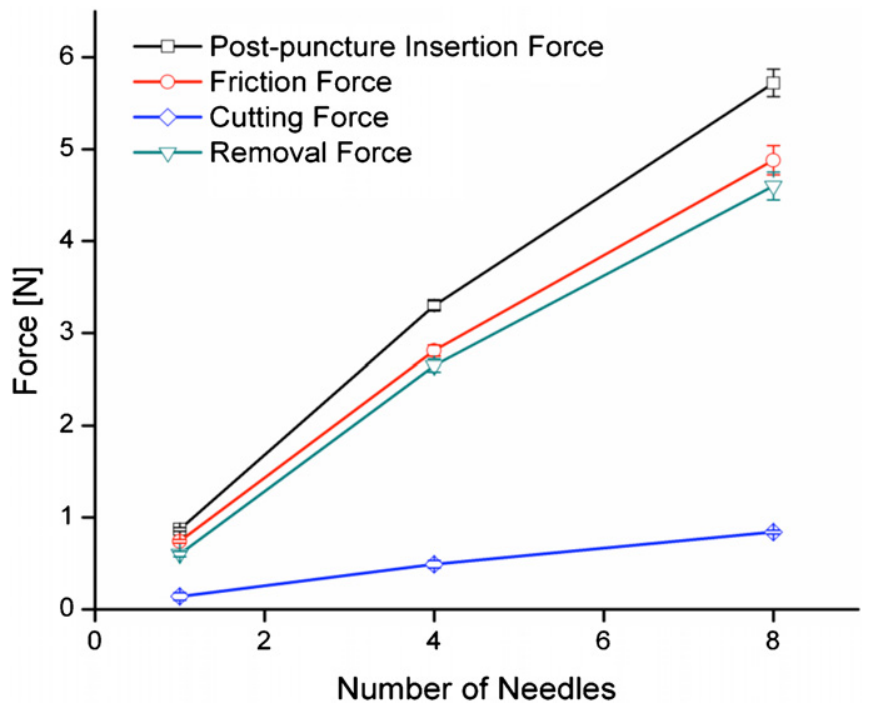

Figure 10. Relationship of post-puncture insertion forces (friction and cutting forces) and removal forces with respect to the number of insertion needles.

overall device robustness as related to the magnitude of force necessary to insert the needle array.

Stiffness force increased with the needle diameter (i.e. decreasing needle gauge). As expected, coring needles were associated with larger stiffness force due to the blunt profile compared to the beveled profile for non-coring needles. Stiffness force could not be determined for needle arrays because the needle tips were not perfectly aligned due to limitations in manual fabrication of the arrays. Therefore, each tip punctured the PDMS sample at slightly different times and a reliable stiffness force could not be determined.

The insertion force, the combination of friction and cutting forces, was also expected to increase with surface area. As surface area increases, frictional force should likewise increase. Frictional force in a $33 \mathrm{G}$ coring needle was larger than that for the non-coring case due to the area contributed by 
Table 2. Summary of failure pressure and failure locations for all septa designs. The arrows indicate failure points.

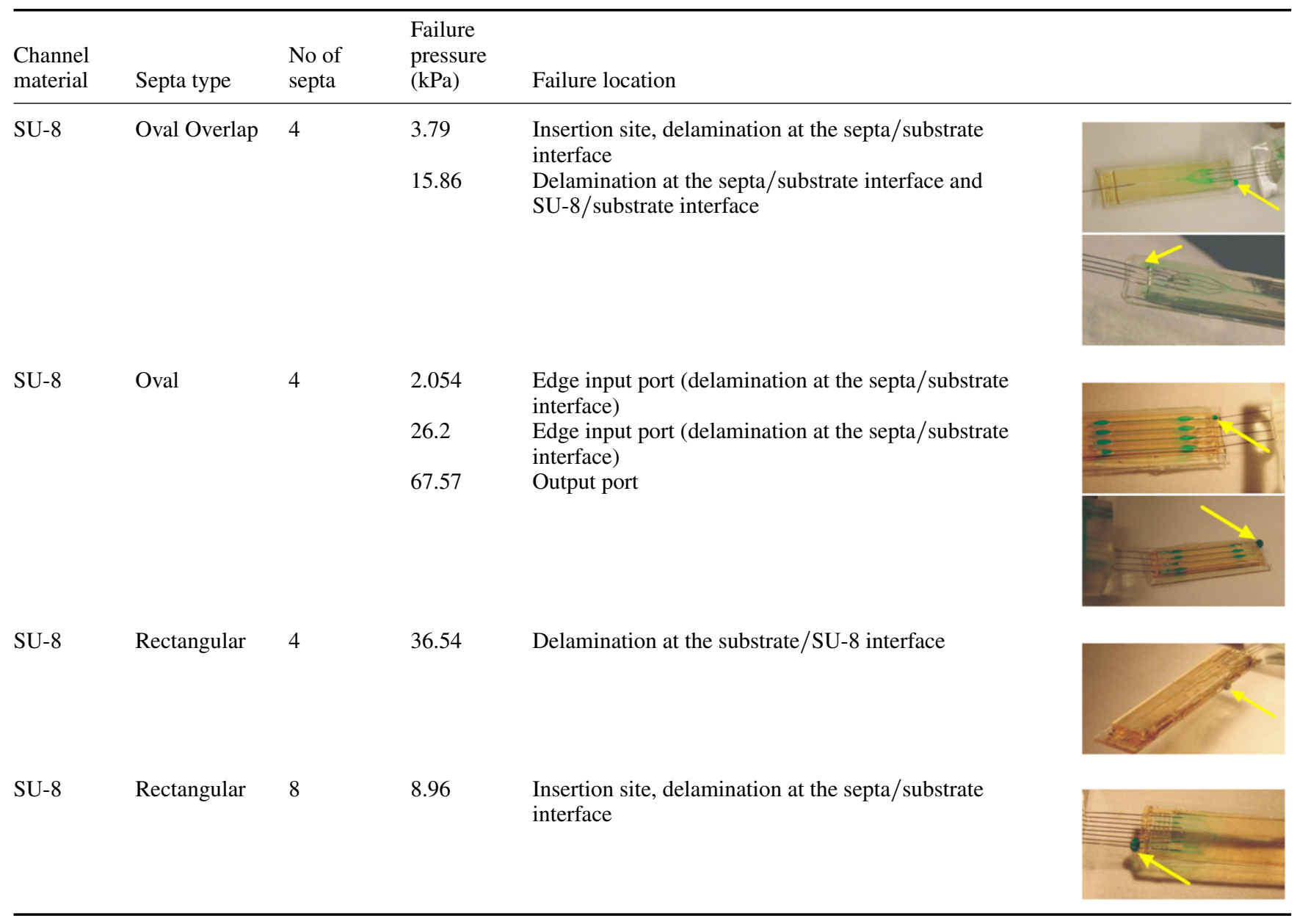

the lumen of the coring needle which was also in contact with the PDMS. Coring needles were also associated with higher cutting force in general due to their blunt profile compared to the beveled tip of the non-coring needle.

Different insertion rates $\left(1\right.$ and $\left.0.5 \mathrm{~mm} \mathrm{~s}^{-1}\right)$ exhibited slight differences in the frictional forces. The lower rate $(0.5$ $\mathrm{mm} \mathrm{s}^{-1}$ ) had a small increase in insertion and removal forces; this may be caused by a change of dynamic frictional force between the needle and PDMS. As expected, the cutting force was not affected.

In all cases, multiple insertions reduced the insertion and removal forces, and therefore affected the sealing capability of the reusable arrayed interconnect. We previously presented information on the sealing capability of a PDMS slab that was punctured multiple times at the same location. As the number of punctures increased, the pressure at which leakage occurred through the puncture site (leakage pressure) decreased. A decrease in sealing ability was attributed to the damage of the PDMS from the repeated insertion and removal damage. However, the relative change in leakage pressure between each additional puncture and removal event decreased with each insertion/ removal event; this suggested a limit to the damage inflicted. The pull-out force of the first puncture for the four-needle array (33G, non-coring), normalized with respect to contact surface area between the needle and septa, was $0.52 \mathrm{~N} \mathrm{~mm}^{-2}$ and decreased to $0.48 \mathrm{~N} \mathrm{~mm}^{-2}$ for the tenth removal event. This result was similar to removal forces of comparable designs of other published reusable connectors, which ranged from 0.08 to $0.95 \mathrm{~N} \mathrm{~mm}^{-2}$ for the first removal event to 0.02 to $0.22 \mathrm{~N} \mathrm{~mm}^{-2}$ for the tenth removal $[8,13,14,17]$.

\subsection{Pressure test}

The leakage pressure associated with each of the three septa designs was determined (table 2). Several factors were identified to explain the wide range of failure pressures for each setpa design. The lower failure pressures for the oval overlap design were attributed to septa delamination from the substrate. During needle array insertion, slight adjustments to align one of the needles induced torque on the other adjacent needles and the septa resulting in delamination of the PDMS from the Parylene-coated substrate. The Parylene coating mitigated thermally induced stress between the SU-8 and glass but did not adhere well to PDMS [30]. Future designs will include an etch step to remove the excess Parylene to allow a direct bond between the PDMS septa and glass substrate.

The distinct septa in the oval design minimized coupling of torque to adjacent needles. Instead, leakage pressure was determined by the weakest needle/septum interface. Following failure of one pair (at $2 \mathrm{kPa}$ ), the septum was resealed using additional PDMS and repressurized. The 
same input location failed again at $26 \mathrm{kPa}$, whereas the other three input locations did not fail. Again, needle alignment affects sealing. The needle guides mitigate the possibility of misalignment of the needle in the horizontal plane; however, small changes in the needle insertion angle translate into increasing angular misalignment the further the needle moves into the septum. If the needle was inserted at a large angle relative to the substrate surface, as opposed to parallel to the substrate surface, then the needle may veer off course and come into contact with the substrate. In this case, the needle is no longer sealed around its circumference by PDMS throughout the entire length of the septum and causes the PDMS to partially delaminate from the substrate near the contact site. This 'tenting' effect creates a potential leakage path along the needle shaft. The third failure point following input resealing was at $67 \mathrm{kPa}$ and located at the output septum. The same factors apply for both input and output interconnects. However, this result indicated that the input septa which did not fail at 2 and $26 \mathrm{kPa}$ of applied pressure were well aligned and tightly sealed.

The robustness of the rectangular septa interconnect was also highly dependent on needle alignment. The SU-8 anchoring posts in this design resulted in a narrow needle path through the septa area. Slight needle misalignment may result in needle tips lodging in the SU-8 posts or, with sufficient insertion force, dislodging of SU-8 posts from the substrate. The four septa rectangular design failed at the device edge. This failure was not related to the interconnect portion of the device, instead indicated poor local adhesion between the SU-8 and Parylene or the SU-8 and substrate. The difference in failure pressure between the four and eight septa arrays was again attributed to manual needle alignment; fewer needles were easier to align and had better performance. The additional force necessary to push eight needles into the septa caused the needles within the array to buckle. The buckling resulted in redirection of the inserting force as torque which in turn caused delamination between the septa and the Parylene. Additionally, several SU-8 posts were dislodged from the substrate, creating additional areas where the septa and substrate were not in contact.

Extended application ( $>24 \mathrm{~h}$ ) of pressurized water was applied to the oval septa design, the most robust design based on the leakage pressure results. The oval design survived continuous application of pressure, $25 \mathrm{kPa}$, without any visible leakage. This result demonstrated the interconnect's ability to be used in applications which require survival in extended pressurized conditions.

\subsection{Parylene microchannel}

Removing the sacrificial photoresist using IPA took approximately 28 days. Acetone is incompatible with photoresist removal in the presence of SU-8 due to delamination [31, 32]. However, dissolution of the photoresist can be accelerated by heating the IPA bath $\left(\sim 50{ }^{\circ} \mathrm{C}\right)$; full dissolution can be achieved in $\sim 20$ days. Later, it was determined that the undiluted photoresist developer (AZ 351) achieved full dissolution within $24 \mathrm{~h}$ with no observable delamination of the SU-8 structures.

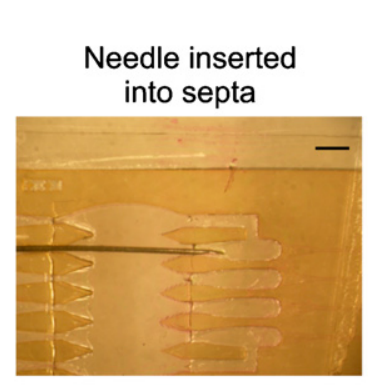

\section{Rhodamine injected, seen traveling into microchannels}

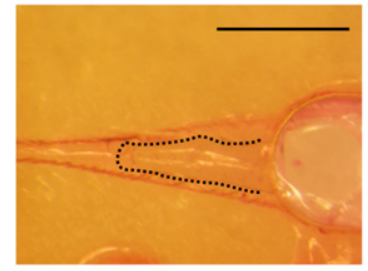

Rhodamine moved into exit port of microchannel

(waterfront is outlined)
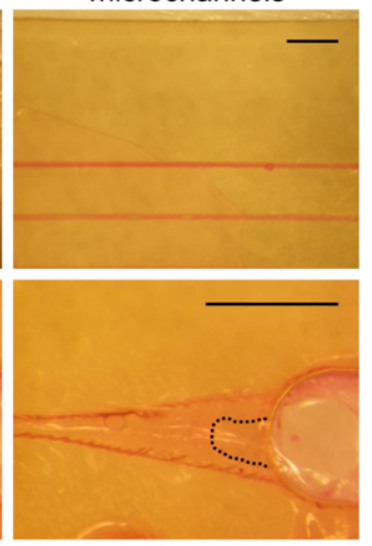

Waterfront of rhodamine continued to move out of microchannel exit

Figure 11. Time lapse images of pressurized Rhodamine B ( $\sim 0.5 \mathrm{psi}, 3.45 \mathrm{kPa})$ moving through a half-packaged microfluidic device with surface micromachined Parylene microchannels. Waterfront is outlined to help identify the liquid/air interface (scale bars represent $1 \mathrm{~mm}$ ).

Complete removal of the sacrificial photoresist was verified optically by capillary wicking of a drop of Rhodamine $\mathrm{B}$ introduced at the channel opening. A half-packaged microfluidic system (inlets only) with Parylene microchannels was also tested. A needle array was inserted into the input septa and Rhodamine B was manually introduced using a syringe ( $\sim 0.5 \mathrm{psi}$ or $\sim 3.45 \mathrm{kPa}$ of pressure was applied) (figure 11).

\subsection{Electrolysis pressure generation}

The flexibility of this interconnect approach and compatibility with microfluidic systems incorporating different features were demonstrated by the inclusion of electrochemical electrodes within interfaced microchannels. Electrolysis actuation was performed and the resulting pressure generated was monitored (figure 12). The electrolysis pressure that can be generated depends on the electrolysis variables (e.g. current, electrode design, electrolyte), the internal volume of the device and volume of air already present in the device or testing setup. The generated pressure within the device was well below the failure pressure of the septa (table 2). Additionally, when the current was removed from the electrodes, recombination of the hydrogen and oxygen gases was observed. Complete recombination of generated gas occurred within $1 \mathrm{~h}$ and no leakage was observed.

\subsection{Sideport functionality}

Sideport functionality was verified by introducing clear liquid stream through the sideport and a dyed liquid stream in the primary access port. Both streams were injected at a rate of $500 \mu \mathrm{L} \mathrm{min} \mathrm{m}^{-1}$. Due to laminar flow characteristics in microfluidic devices, distinct and adjacent streams were 


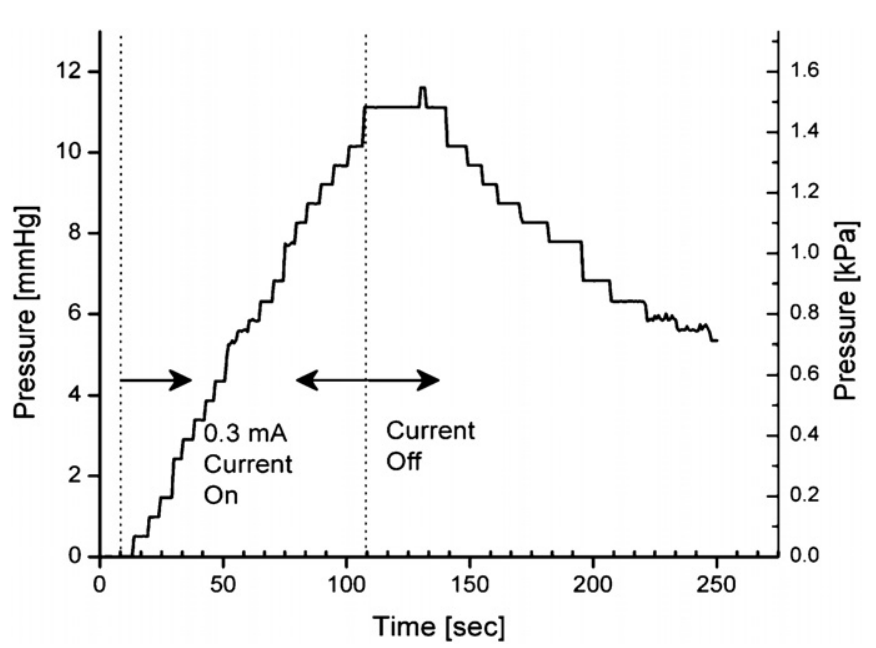

Figure 12. Internal pressure change following electrolysis actuation. Pressure increases when current $(0.3 \mathrm{~mA})$ is applied to the interdigitated electrodes, and pressure decreases when current is turned off and the oxygen and hydrogen gas recombine into water.

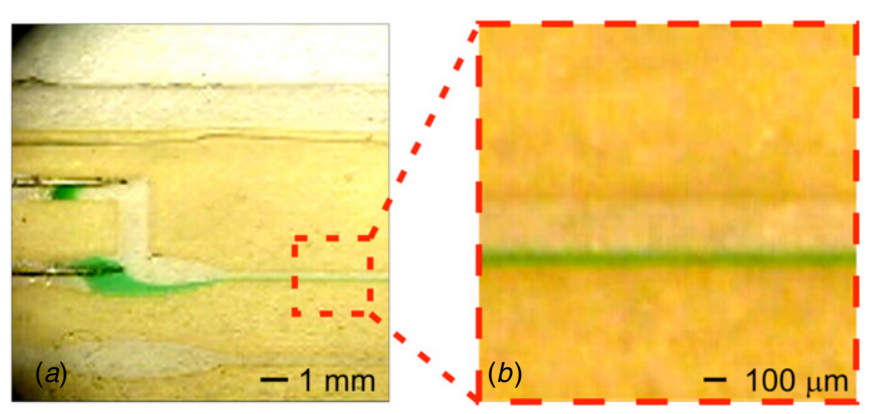

Figure 13. Time lapse images of the sideport function: $(a)$ dyed water introduced in the main septum and un-dyed water through the sideport, $(b)$ close-up image of the laminar flow within the microchannel.

observed in the channel (figure 13). More sideports for multiple inputs into a single microchannel can be added but at the expense of chip real estate.

\section{Discussion}

The arrayed septa and needle design allows for multiple simultaneous macro-to-micro connections to be established in a microfluidic device. PDMS is an excellent choice as a septa material. The compliant nature of PDMS is beneficial for three reasons: (1) it allows the septa to deform around the needle, providing a reliable and robust seal suitable for multiple uses; (2) the septa material displaced during insertion returns to the original state after the needles are removed, sealing access to the device interior; and (3) it allows densely packed and properly aligned needles to be inserted simultaneously without insertion stresses interfering with neighboring needles.

FEM results showed that needle insertion induced stress within the septa dissipates exponentially with respect to distance from the needle shaft. The stress decayed to $36.8 \%$ of the maximum value within $60 \mu \mathrm{m}$. To prevent the stress from extending to the SU-8 anchor, a minimum distance between the needle shaft and SU-8 housing of $120 \mu \mathrm{m}$ is recommended. The rectangular septa design, the one having the smallest spacing between the needle and SU-8 anchor, had a septum width of $500 \mu \mathrm{m}$. Insertion of a $33 \mathrm{G}$ needle with an outer diameter of $203 \mu \mathrm{m}$ left approximately $150 \mu \mathrm{m}$ of PDMS between the needle exterior and SU-8 anchor components. Denser rectangular septa are possible; however, additional space between the needle wall and SU-8 anchor may be prudent to account for any deviations in the needle path (i.e. needle misalignment) from the septa center.

As shown in the insertion and removal force tests, force scaled linearly with the number of needles and decreased for needles with (1) smaller diameters and (2) non-coring tips. The number of needles in an array can be maximized by using the smallest diameter non-coring needles possible. However, the needles must possess adequate rigidity to penetrate the PDMS septa without buckling which may lead to needle misalignment and interconnect failure.

Needle misalignment, leading to septa/substrate delamination, was the main cause of failure. Needle guides in the SU-8 housing helped ensure that the needles were inserted at the center of the septa; however, the needle guides only provided lateral alignment and were not sufficient to keep the needles parallel to the substrate. Furthermore, Okamura et al demonstrated that needle bending is more prevalent for beveled tip needles [33]. Beveled needles are necessary to minimize insertion and removal forces, therefore, maximizing the reusability of this interconnect. Additionally, commercial bevel-tipped needles are readily available. Therefore, other methods to improve needle alignment need to be implemented.

Needle misalignment can be mitigated by improving needle stiffness; however, trade-offs need to be considered. The needles used to establish the microfluidic connections were $2.54 \mathrm{~cm}$ in length and the septa were $4-4.5 \mathrm{~mm}$ in length. The needle length can be shortened to better match the septa length; however, the needle length determines the maximum length of the septa which is linked to needle removal force. Removal force is an indicator of the maximum leakage pressure the septa can withstand. A smaller needle gauge (i.e. larger diameter) may be used. Larger diameter needles require greater insertion force and greater septa thicknesses to accommodate the increased diameter. Finally, the needle guides can be altered (e.g. lengthened) to help establish and maintain the needle position and absorb any buckling effect, but lengthening the needle guides increases the device footprint. Thus, many tradeoffs exist and must be carefully considered when designing interconnects. Also, greater control of septa and needle alignment is possible with precision commercial manufacturing and may mitigate some of the factors observed here.

Septa delamination from the Parylene or the SU-8 was also a common failure mode. Bonding between the septa to the SU8 anchor and substrate package can be improved. First, the Parylene can be removed using oxygen plasma from the area outlined by the SU-8 anchor prior to adding the SU-8 layer. PDMS has a higher bond strength to glass than Parylene. The SU-8 sidewalls can also be roughened to increase the contact surface area.

The sideport allowed the injection of two distinct fluids into a single microchannel. However, the sideport feature 
can be extended to allow multiple inputs into the same microchannel, thus providing a modular design which uses none, some, or all of the sideports. Sideports can also be placed perpendicular to the microchannel; these sideports would allow samples to be drawn from any location along the fluidic stream, or for the introduction of sensors into the microchannel.

\section{Conclusion}

An arrayed interconnect design capable of rapid and multiple simultaneous connections without the use of adhesives to a microfluidic device is presented. The interconnects are reusable and were proven to allow at least ten complete uses where connections were established, broken and reestablished. Interconnect design parameters were explored. $33 \mathrm{G}$ non-coring needles provided adequate strength for insertion while minimizing SU-8 septa housing thickness. The linear relationship between insertion and removal forces and the number of needles was theoretically and experimentally verified. While the failure pressure of the arrayed interconnect was limited by the weakest point, up to $62 \mathrm{kPa}$ of pressure was supported. Also, interconnects were able to maintain $25 \mathrm{kPa}$ of pressure for over $24 \mathrm{~h}$. Connector spacings of 1 and $2.54 \mathrm{~mm}$ were fabricated; however, the FEM analysis of stress distribution shows that $33 \mathrm{G}$ needles can be spaced as close as $443 \mu \mathrm{m}$, center-to-center. Functionality of additional features such as Parylene microchannels, needle guides, sideports for combining two fluids and electrolysis structures was shown.

The arrayed interconnect method is advantageous over current methods because it (1) can be reused if necessary, (2) has a horizontal orientation, which increases the connection robustness without the need for adhesives and does not interfere with visual or microscope observations, and (3) establishes multiple connections simultaneously, reducing the time. Additionally, it is suitable for a standard method for microfluidic connections, thereby simplifying microfluidic packages.

\section{Acknowledgments}

This work was funded in part by the Engineering Research Centers Program of the National Science Foundation (NSF) under Award Number EEC-0310723 and an NSF CAREER grant under Award Number EEC-0547544. The authors would like to thank Dr Donghai Zhu and other members of the Biomedical Microsystems Lab at the University of Southern California for their contributions to this work.

\section{References}

[1] Ho C M and Tai Y C 1998 Micro-electro-mechanical-systems (MEMS) and fluid flows Annu. Rev. Fluid Mech. 30 579-612

[2] Whitesides G M 2006 The origins and the future of microfluidics Nature 442 368-73

[3] Fredrickson C K and Fan Z H 2004 Macro-to-micro interfaces for microfluidic devices Lab Chip 4 526-33
[4] Lo R and Meng E 2009 Macro-to-micro fluidic interfacing Lab-on-a-Chip Technology: vol 1. Fabrication and Microfluidics ed K E Herold and A Rasooly (Norfolk, UK: Caister Academic Press) chapter 22 pp 353-70

[5] Anderson J R, Chiu D T, Jackman R J, Cherniavskaya O, McDonald J C, Wu H K, Whitesides S H and Whitesides G M 2000 Fabrication of topologically complex three-dimensional microfluidic systems in PDMS by rapid prototyping Anal. Chem. 72 3158-64

[6] Bhagat A A S, Jothimuthu P, Pais A and Papautsky I 2007 Re-usable quick-release interconnect for characterization of microfluidic systems J. Micromech. Microeng. 17 42-9

[7] Christensen A M, Chang-Yen D A and Gale B K 2005 Characterization of interconnects used in PDMS microfluidic systems J. Micromech. Microeng. 15 928-34

[8] Li S F and Chen S C 2003 Polydimethylsioxane fluidic interconnects for microfluidic systems IEEE Trans. Adv. Packag. 26 242-7

[9] Meng E, Wu S and Tai Y-C 2001 Silicon couplers for microfluidic applications Fresenius' J. Anal. Chem. $371270-5$

[10] Murphy E R, Inoue T, Sahoo H R, Zaborenko N and Jensen K F 2007 Solder-based chip-to-tube and chip-to-chip packaging for microfluidic devices Lab Chip 7 1309-14

[11] Pattekar A V and Kothare M V 2003 Novel microfluidic interconnectors for high temperature and pressure applications J. Micromech. Microeng. 13 337-45

[12] Yang Z and Maeda R 2003 Socket with built-in valves for the interconnection of microfluidic chips to macro constituents J. Chromatogr. A 1013 29-33

[13] Yao T-J, Lee S, Fang W and Tai Y-C 2000 Micromachined rubber O-ring micro-fluidic couplers Proc. IEEE Micro Electro Mechanical Systems (MEMS) pp 624-7

[14] Chiou C H and Lee G B 2004 Minimal dead-volume connectors for microfluidics using PDMS casting techniques J. Micromech. Microeng. 14 1484-90

[15] Lee E S, Howard D, Liang E, Collins S D and Smith R L 2004 Removable tubing interconnects for glass-based micro-fluidic systems made using ECDM J. Micromech. Microeng. 14 535-41

[16] Puntambekar A and Ahn C H 2002 Self-aligning microfluidic interconnects for glass- and plastic-based microfluidic systems J. Micromech. Microeng. 12 35-40

[17] Lo R and Meng E 2008 Integrated and reusable in-plane microfluidic interconnects Sensors Actuators B 132 531-9

[18] Lo R and Meng E 2008 Characterization and fabrication of high-density, on-demand, reusable, in-plane polymer interconnects towards standardized microfluidic packaging 12th Int. Conf. on Miniaturized Systems for Chemistry and Life Sciences ( $\mu$ TAS) pp 709-11

[19] MicroChem Permanent Epoxy Negative Photoresist-Processing Guidelines for: SU-8 2100 and $S U-82150$ www.microchem.com

[20] Yao T J, Yang X and Tai Y C 2002 BrF3 dry release technology for large freestanding parylene microstructures and electrostatic actuators Sensors Actuators A 97-8 771-5

[21] Tas N, Sonnenberg T, Jansen H, Legtenberg R and Elwenspoek M 1996 Stiction in surface micromachining J. Micromech. Microeng. 6 385-97

[22] Li P Y, Shih J, Lo R, Saati S, Agrawal R, Humayun M S, Tai Y C and Meng E 2008 An electrochemical intraocular drug delivery device Sensors Actuators A 143 41-8

[23] Lo R and Meng E 2007 A reusable in-plane polymer integrated microfluidic interconnect Solid-State Sensors, Actuators and Microsystems Conf. pp 2067-70

[24] Rodger D C et al 2008 Flexible parylene-based multielectrode array technology for high-density neural stimulation and recording Sensors Actuators B 132 449-60 
[25] Lo R 2009 Modular bio-microelectromechanical systems (bioMEMS): intraocular drug delivery device and microfluidic interconnects PhD Dissertation University of Southern California

[26] Timoshenko S and Goodier J N 1970 Theory of Elasticity (New York: McGraw-Hill) p 567

[27] Abolhassani N, Patel R and Moallem M 2007 Needle insertion into soft tissue: a survey Med. Eng. Phys. 29 413-31

[28] Okamura A M, Simone C and O'Leary M D 2004 Force modeling for needle insertion into soft tissue IEEE Trans. Biomed. Eng. 51 1707-16

[29] Gent A N and Liu G L 1991 Pull-out and fragmentation in model fiber composites J. Mater. Sci. 26 2467-76
[30] Lo R, Li P-Y, Saati S, Agrawal R, Humayun M and Meng E 2008 A passive MEMS drug delivery pump for treatment of ocular diseases Biomed. Microdevices 11 959-70

[31] Agarwal M, Gunasekaran R A, Coane P and Varahramyan K 2005 Scum-free patterning of SU-8 resist for electroforming applications J. Micromech. Microeng. 15 130-5

[32] Miserendino S 2005 A modular microfluidic approach to nano high-performance liquid chromatography with electrochemical detection PhD Dissertation California Institute of Technology

[33] Okamura A M, Simone C and O'Leary M D 2004 Force modeling for needle insertion into soft tissue IEEE Trans. Biomed. Eng. 51 1707-16 